\title{
Plasma C1q/TNF-Related Protein-3 (CTRP-3) and High-Mobility Group Box-1 (HMGB-1) Concentrations in Subjects with Prediabetes and Type 2 Diabetes
}

\author{
Huili Wei, Hua Qu, Hang Wang, and Huacong Deng \\ Department of Endocrinology, The First Affiliated Hospital of Chongqing Medical University, Chongqing 400016, China \\ Correspondence should be addressed to Huacong Deng; deng huacong@126.com
}

Received 13 April 2016; Revised 28 August 2016; Accepted 30 August 2016

Academic Editor: Andrea Tura

Copyright (C) 2016 Huili Wei et al. This is an open access article distributed under the Creative Commons Attribution License, which permits unrestricted use, distribution, and reproduction in any medium, provided the original work is properly cited.

\begin{abstract}
Aims. To detect the association of Clq/TNF-related protein-3 (CTRP-3) and high-mobility group box-1 (HMGB-1) in subjects with prediabetes (pre-DM) and newly diagnosed type 2 diabetes (nT2DM). Methods. 224 eligible participants were included. The $75 \mathrm{~g}$ oral glucose tolerance test (OGTT) and several clinical parameters of metabolic disorders and cytokines were measured. All participants were divided into three groups: normal glucose tolerance (NGT, $n=62)$, pre-DM $(n=111)$, and nT2DM group $(n=56)$. Results. Plasma CTRP-3 concentrations were significantly lower in subjects with pre-DM and nT2DM than that of the NGT group, while plasma HMGB-1 levels were higher in pre-DM and nT2DM group compared with the NGT group $(P<0.05)$. A multiple linear regression analysis showed both plasma CTRP-3 and HMGB-1 concentrations were independently associated with homeostasis model assessment for insulin resistance (HOMA-IR) and interleukin-6 (IL-6) $(P<0.05$ for all). Further multiple logistical regression analyses revealed that both plasma CTRP-3 and HMGB-1 levels were significantly associated with pre-DM and nT2DM after adjusting for several confounders ( $P<0.001$ for all). Conclusions. Circulating CTRP-3 and HMGB- 1 concentrations might be promising biomarkers to predict prediabetes and type 2 diabetes.
\end{abstract}

\section{Introduction}

Since the dramatic changes in lifestyle worldwide, diabetes has become one of the most common metabolic disorders globally. In 2013, the International Diabetes Federation (IDF) reported 382 million people with diabetes and the number was projected to reach 592 million by 2035 [1]. Of note, another piece of data from IDF also revealed $45.8 \%$ or 174.8 million of undiagnosed diabetes in all diabetes cases globally [2]. Prediabetes (pre-DM) is defined as either fasting or postchallenge glycemia, including impaired fasting glucose (IFG), impaired glucose tolerance (IGT), and combined IFG and IGT (impaired glucose regulation, IGR) [3]. As an intermediate state between normal glucose tolerance (NGT) and type 2 diabetes (T2DM), pre-DM is characterized by insulin resistance and impaired insulin secretion $[4,5]$. Besides, the chronic low-grade inflammation also contributes to the progression of pre-DM to T2DM [6]. Though most subjects with pre-DM remain clinically asymptomatic, the micro- and macrovascular complications of diabetes already exist in this stage [7]. Recent data have showed that pre-DM is closely associated with poor cardiovascular outcomes [8]. IFG was also found to be correlated with unrecognized myocardial infarctions in patients without fundamental cardiovascular disease [9]. It is estimated that up to $70 \%$ of individuals with pre-DM will progress to diabetes at a rate of $5-10 \%$ per year [7]. In China, the age-adjusted prevalence of pre-DM is $15.5 \%$, accounting for 148.2 million people with pre-DM [10].

The Clq/tumor necrosis factor- (TNF-) related protein3 (CTRP-3) belongs to the CTRP family which shares a highly conserved paralog of adiponectin [11]. CTRP-3 is abundantly expressed in the adipose tissue, kidney, uterus, and testis in adult animals [12]. It is well established that CTRP-3 plays an important role in modulating the hepatic glucose and lipid metabolism $[13,14]$. In normal C57BL/6 and leptin-deficient ob/ob mice, recombinant CTRP-3 injection displayed a significant antidiabetic effect [13]. Recent clinical studies demonstrate that circulating CTRP-3 is associated 
with T2DM and obesity $[15,16]$. However, conflicting results remain between circulating CTRP-3 and T2DM. Choi et al. [15] reported increased circulating levels of CTRP-3 in subjects with pre-DM and T2DM, while other groups showed lower levels of circulating CTRP-3 in newly diagnosed T2DM patients $[16,17]$. Furthermore, CTRP-3 is considered an anti-inflammatory cytokine. In a high-fat fed transgenic mouse model, CTRP-3 mitigated systemic inflammation in the context of obesity and insulin resistance [18]. It has been reported that CTRP-3 could reduce the secretion of interleukin-6 (IL-6) and tumor necrosis factor- $\alpha$ (TNF$\alpha$ ) by suppressing the nuclear factor- $\kappa \mathrm{B}(\mathrm{NF}-\kappa \mathrm{B})$ signaling [19].

High-mobility group box-1 (HMGB-1) was first discovered in 1973 [20]. It was originally regarded as an important factor for maintaining the structure and stability of the chromosome [20]. So far, several studies have revealed that HMGB-1 also exerts extracellular functions via HMGB1 related receptors. For instance, exogenous HMGB-1 can promote the release of proinflammatory cytokines by binding to their receptors such as RAGE, TLR2, and TLR4 [21]. In addition, several studies indicate that HMGB-1 is related to insulin resistance [22], obesity, and T2DM [23]. Wang et al. [23] found plasma HMGB-1 levels were increased in subjects with T2DM and/or obesity. Chen et al. [24] found HMGB1 was elevated in both T2DM patients and high-glucose cultured SV40 MES 13 cells.

Though previous studies have reported the role of circulating CTRP-3 and HMGB-1 in T2DM and obesity, there is little information concerning the associations of circulating CTRP-3 and HMGB-1 with pre-DM. Pre-DM is a high risk factor of cardiovascular events and plays an important role in the development of diabetes, so studies on biomarkers to predict pre-DM deserve further investigation. In this study, we aim to explore the association of plasma CTRP-3 and HMGB-1 with newly diagnosed pre-DM and T2DM.

\section{Methods}

2.1. Study Design and Subjects. A total of 420 subjects (age ranged from 40 to 75 ) with newly diagnosed T2DM (nT2DM) or pre-DM or healthy controls were recruited from the outpatients of The First Affiliated Hospital of Chongqing Medical University between June 2014 and October 2015. After the $75 \mathrm{~g}$ oral glucose tolerance test (OGTT) and routine biochemical check-up, a total of 224 eligible participants were included (the exclusion criteria were presented in Supplemental Figure 1 in Supplementary Material available online at http://dx.doi.org/10.1155/2016/9438760). All participants were divided into normal glucose tolerance group (NGT, $n=$ $62)$, pre-DM group $(n=111)$, and nT2DM group $(n=$ 56). The diagnosis of pre-DM and nT2DM was based on the ADA criteria [25]. All enrolled participants have not received any hypoglycemic agents, diet-control, or exercise prior to screening. Present study was in accordance with the Declaration of Helsinki of the World Medical Association and was approved by the Ethics Committee of The First Affiliated Hospital of Chongqing Medical University,
Chongqing, China. Written informed consent was obtained from all participants.

2.2. Anthropometric, Clinical, and Demographic Measurements. Height, body weight, waist circumference, and hip circumference were measured by standardized methods in all subjects. Blood pressure was measured using an automated electronic device (OMRON Model HEM-725 FUZZY, Omron Company, Dalian, China) three times consecutively after a 5-minute rest; the three readings were averaged for further analyses. Blood samples were collected in the morning (8-10 a.m.) after an overnight fasting ( $\geq 8$ hours). Plasma samples were obtained by centrifugation at $2000 \times \mathrm{g}$ for 10 minutes at $4^{\circ} \mathrm{C}$ and were kept at $-80^{\circ} \mathrm{C}$ before analyses. All analyses were performed within one month. Plasma glucose levels were measured using the glucose oxidase method, while $\mathrm{HbAlc}$ was measured using the high-performance liquid chromatography (VARIANTTM II and D-10TM Systems, BIO-RAD, USA). Fasting insulin (FINS) was measured using an autoanalyzer (ARCHITECT i2000SR System, Abbott Laboratories, USA). Lipid profiles such as triglyceride (TG), total cholesterol (TC), high-density lipoprotein cholesterol (HDL-C), and low-density lipoprotein cholesterol (LDL-C) were assayed by enzymatic methods, while the liver and kidney functions were determined using a biochemical aut-analyzer (ARCHITECT c16000 System, Abbott Laboratories, IL, USA).

The body mass index (BMI) formula is computed as weight in kilograms divided by height in meters squared. The waist-to-hip ratio (WHR) formula is the waist circumference in centimeters divided by the hip circumference in centimeters. The homeostasis model assessment of insulin resistance $(\mathrm{HOMA}-\mathrm{IR})=$ fasting insulin $(\mathrm{mU} / \mathrm{L}) *$ fasting plasma glucose $(\mathrm{mmol} / \mathrm{L}) / 22.5$.

2.3. Assessment of Plasma CTRP-3, HMGB-1, and IL-6 Levels. Plasma CTRP-3, HMGB-1, and IL-6 levels were determined by commercial ELISA kits according to the manufacturers' instructions (Human ELISA kit, Uscn Life Science Inc., Wuhan, China). The intra-assay coefficients of variation were less than $8 \%$, while the interassay coefficients of variation were less than $10 \%$. All the assays were carried out in duplicate, and no significant cross-reactivity or interference was observed.

2.4. Statistical Analyses. The SPSS software (IBM, Armonk, NY, version 19.0) was used for all statistical analyses. Data are presented as means \pm standard deviation. Nonnormally distributed parameters including LDL-C and $\gamma$-GGT were logarithmically transformed before analyses. Comparisons between groups were performed using the analysis of variance (ANOVA). Interrelationships between variables were assessed using a partial correlation analysis by controlling for age and gender. The distributions of plasma CTRP-3 $(<395$, $395 \sim 451$, and $>455, \mathrm{ng} / \mathrm{mL})$ and HMGB-1 $(<4.76,4.76 \sim 5.38$, and $>5.38, \mathrm{ng} / \mathrm{mL}$ ) were further divided into tertiles, and the linear trend was estimated by a linear-by-linear association of the chi-square test. A multivariate linear regression was 
TABLE 1: Clinical and biochemical characteristics of the study subjects.

\begin{tabular}{|c|c|c|c|c|c|}
\hline Variables & NGT & Pre-DM & nT2DM & $F$ value & $P$ value \\
\hline Participants (M/F) & $62(30 / 32)$ & $111(55 / 56)$ & $56(27 / 29)$ & & \\
\hline Age (years) & $52.7 \pm 5.6$ & $53.6 \pm 5.5$ & $56.6 \pm 8.05^{* \dagger}$ & 6.399 & 0.002 \\
\hline $\mathrm{FPG}(\mathrm{mmol} / \mathrm{L})$ & $5.0 \pm 0.3$ & $6.4 \pm 0.3^{*}$ & $7.9 \pm 0.7^{* \dagger}$ & 72.24 & $<0.001$ \\
\hline INS & $5.5 \pm 0.9$ & $5.9 \pm 0.9^{*}$ & $7.3 \pm 1.2^{* \dagger}$ & 37.63 & $<0.001$ \\
\hline HOMA-IR & $1.09 \pm 0.17$ & $1.71 \pm 0.11^{*}$ & $2.69 \pm 0.39^{* \dagger}$ & 52.08 & $<0.001$ \\
\hline $2 \mathrm{~h} \mathrm{PG}(\mathrm{mmol} / \mathrm{L})$ & $6.8 \pm 0.6$ & $9.3 \pm 1.3^{*}$ & $13.1 \pm 1.6^{* \dagger}$ & 25.17 & $<0.001$ \\
\hline HbAlc (\%) & $4.9 \pm 0.6$ & $6 \pm 0.2^{*}$ & $7.6 \pm 0.6^{* \dagger}$ & 63.21 & $<0.001$ \\
\hline BMI $\left(\mathrm{kg} / \mathrm{m}^{2}\right)$ & $22.42 \pm 1.78$ & $22.97 \pm 2.04$ & $23.84 \pm 2.05^{* \dagger}$ & 7.663 & 0.001 \\
\hline WHR & $0.83 \pm 0.06$ & $0.85 \pm 0.05^{*}$ & $0.88 \pm 0.03^{* \dagger}$ & 21.486 & $<0.001$ \\
\hline SBP (mmHg) & $128 \pm 13$ & $129 \pm 14$ & $132 \pm 12$ & 1.19 & 0.306 \\
\hline DBP (mmHg) & $77 \pm 7$ & $77 \pm 10$ & $79 \pm 9$ & 2.268 & 0.106 \\
\hline TC (mmol/L) & $4.51 \pm 0.6$ & $4.54 \pm 0.72$ & $4.77 \pm 0.61^{* \dagger}$ & 2.85 & 0.06 \\
\hline $\mathrm{TG}(\mathrm{mmol} / \mathrm{L})$ & $1.12 \pm 0.5$ & $1.36 \pm 0.65^{*}$ & $1.6 \pm 0.79^{* \dagger}$ & 7.968 & $<0.001$ \\
\hline LDL-C $(\mathrm{mmol} / \mathrm{L})^{\#}$ & $0.77 \pm 0.26$ & $0.84 \pm 0.28$ & $0.92 \pm 0.16^{* \dagger}$ & 5.182 & 0.006 \\
\hline HDL-C (mmol/L) & $1.4 \pm 0.34$ & $1.24 \pm 0.28^{*}$ & $1.04 \pm 2.06^{* \dagger}$ & 12.163 & $<0.001$ \\
\hline $\operatorname{ALT}(\mathrm{U} / \mathrm{L})$ & $20 \pm 6$ & $20 \pm 8$ & $23 \pm 10$ & 1.68 & 0.189 \\
\hline AST (U/L) & $22 \pm 8$ & $23 \pm 7$ & $24 \pm 7^{*}$ & 2.562 & 0.079 \\
\hline$\gamma$-GGT $(\mathrm{U} / \mathrm{L})^{\#}$ & $2.78 \pm 0.38$ & $3.07 \pm 0.55^{*}$ & $3.42 \pm 0.65^{* \dagger}$ & 21.01 & $<0.001$ \\
\hline $\mathrm{Cr}$ & $63 \pm 8$ & $63 \pm 14$ & $64 \pm 8$ & 0.397 & 0.673 \\
\hline IL-6 (pg/mL) & $47.4 \pm 7.1$ & $59.44 \pm 6.79^{*}$ & $77.58 \pm 4.03^{* \dagger}$ & 39.46 & $<0.001$ \\
\hline
\end{tabular}

Data were expressed as means \pm standard deviation. Nonnormally distributed parameters including LDL-C and $\gamma$-GGT were logarithmically transformed before analyses. Differences between multiple groups were tested by analysis of variance (ANOVA) for continuous variables. NGT, normal glucose tolerance; pre-DM, prediabetes states (including IFG, impaired fasting glucose; IGT, impaired glucose tolerance; IGR, impaired glucose regulation); T2DM, type 2 diabetes mellitus; M, male; F, female; FPG, fasting plasma glucose; FINS, fasting serum insulin; HOMA-IR, homeostasis model assessment for insulin resistance; $2 \mathrm{~h}$ PG, $2 \mathrm{~h}$ postchallenge plasma glucose; HbAlc, hemoglobin Alc; BMI, body mass index; WHR, waist-to-hip ratio; SBP, systolic blood pressure; DBP, diastolic blood pressure; TC, total cholesterol; TG, triglyceride; LDL-C, low-density lipoprotein cholesterol; HDL-C, high-density lipoprotein cholesterol; AST, aspartate transaminase; ALT, alanine transaminase; $\gamma$-GGT, gamma-glutamyl transpeptidase; IL-6, interleukin-6.

${ }^{\#}$ Logarithmically transformed variables.

${ }^{*} P<0.05$ compared with $\mathrm{NGT},{ }^{\dagger} P<0.05$ compared with pre-DM.

performed to identify the risk factors of plasma CTRP-3 and HMGB-1. Associations among plasma CTRP-3 and HMGB1 levels and pre-DM and nT2DM were examined using the multiple logistic regression analyses. $P$ values $<0.05$ were considered statistically significant.

\section{Results}

3.1. Characteristics of the Study Subjects. The main characteristics of the subjects are presented in Table 1. Subjects in nT2DM group were older than those of the NGT and pre-DM group $(P<0.05)$. Compared with the NGT group, subjects in the pre-DM and nT2DM group exhibited higher levels of HOMA-IR, WHR, TG, $\gamma$-GGT, and IL-6, while the levels of HDL-C in the NGT group were higher than those of the preDM and nT2DM group (all $P<0.05$ ). Besides, subjects in nT2DM group had higher levels of BMI, TC, and LDL-C compared with the pre-DM group (all $P<0.05$ ). There were no significant differences in gender, SBP, DBP, ALT, AST, and creatinine distributions among the three groups $(P>0.05)$.

3.2. Plasma CTRP-3 and HMGB-1 Levels and Their Associations with Anthropometric and Biochemical Parameters. As shown in Figures $1(\mathrm{c})$ and $1(\mathrm{~d})$, there were no significant differences in plasma CTRP-3 ([420.39 \pm 64.16$]$ versus [432.14 \pm $65.12], \mathrm{ng} / \mathrm{mL})$ and HMGB-1 $([5.13 \pm 0.65]$ versus [5.22 \pm $0.78], \mathrm{ng} / \mathrm{mL}$ ) concentrations between men and women (all $P>0.05)$. Compared with the NGT group (486.548 \pm $37.09, \mathrm{ng} / \mathrm{mL}$ ), subjects displayed a decreased trend of plasma CTRP-3 concentrations in pre-DM $(419.649 \pm 46.34, \mathrm{ng} / \mathrm{mL})$ and nT2DM group $(356.107 \pm 49.5, \mathrm{ng} / \mathrm{mL}$ ) (Figure $1(\mathrm{a}), P$ for trend $<0.001$ ). Contrary to CTRP-3, plasma HMGB-1 showed an increased trend across the NGT $(4.67 \pm 0.37, \mathrm{ng} / \mathrm{mL})$, pre$\mathrm{DM}(5.04 \pm 0.5, \mathrm{ng} / \mathrm{mL})$, and $\mathrm{nT} 2 \mathrm{DM}$ group $(5.9 \pm 0.81, \mathrm{ng} / \mathrm{mL})$ (Figure 1(b), $P$ trend $<0.001$ ).

Then, plasma CTRP-3 levels were divided into tertiles, including the low $(<396, \mathrm{ng} / \mathrm{mL})$, medium $(398 \sim 451, \mathrm{ng} / \mathrm{mL})$, and high group $(>455, \mathrm{ng} / \mathrm{mL})$. Similarly, the tertiles of plasma HMGB-1 were divided as the low $(<4.76, \mathrm{ng} / \mathrm{mL})$, medium $(4.76 \sim 5.38, \mathrm{ng} / \mathrm{mL})$, and high group $(>5.38, \mathrm{ng} / \mathrm{mL})$. The presence of pre-DM was $53.9,77.2$, and $43.2 \%$, respectively, across the HMGB-1 tertiles $(X 2=3.108, P$ trend $=$ 0.026 ), whereas the presence of nT2DM was 10.5, 12.7, and $54.1 \%$, respectively $(X 2=44.07, P$ trend $<0.001)$. Besides, the proportion of pre-DM was $41,73.3$, and $30.3 \%$, respectively, across the tertiles of CTRP-3 $(X 2=16.424, P$ trend $<0.001)$, 


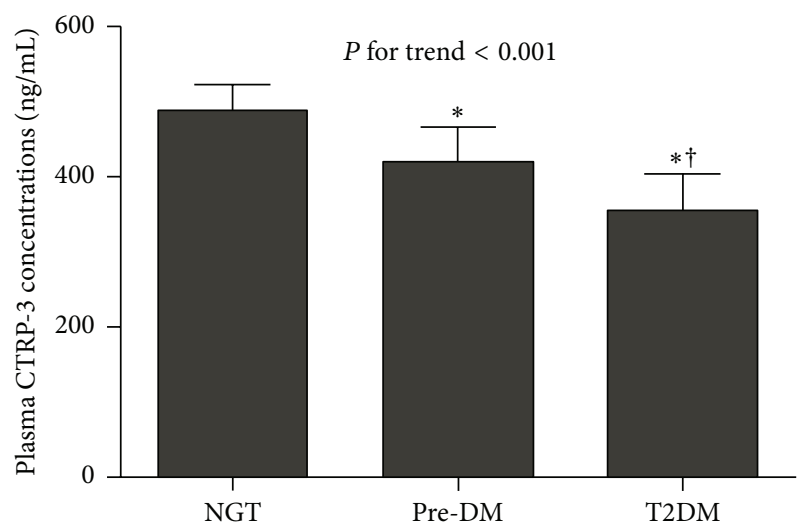

(a)

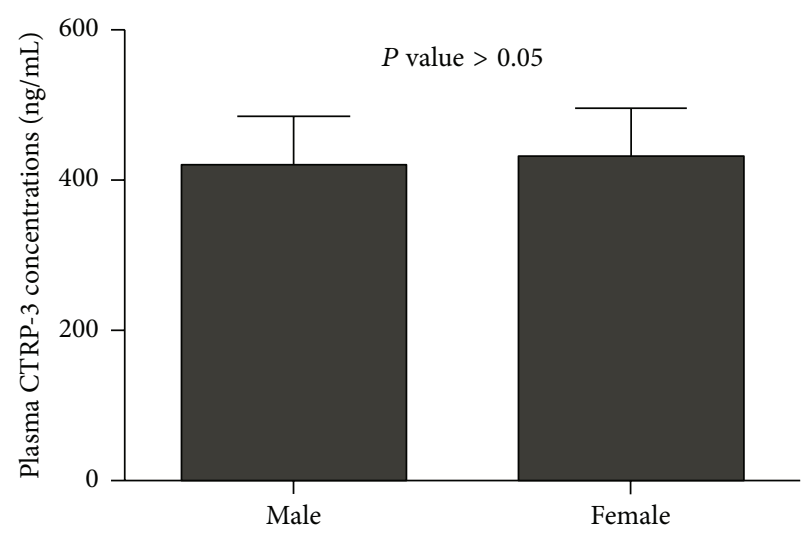

(c)

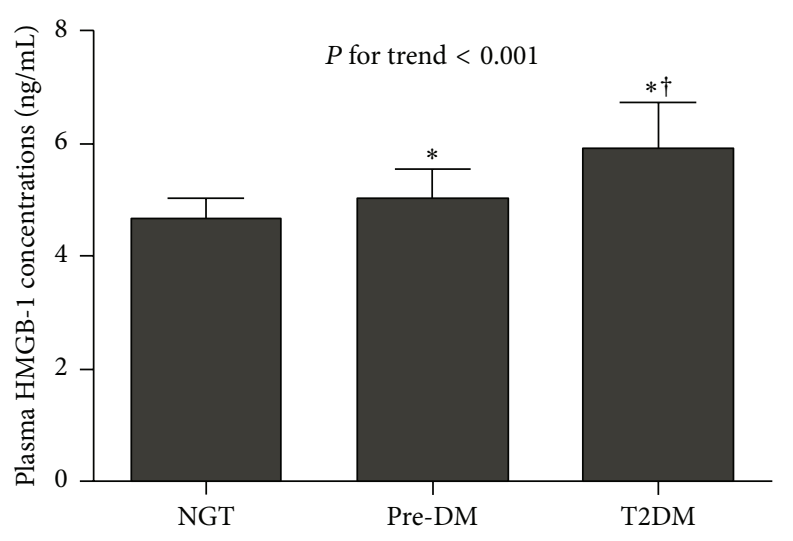

(b)

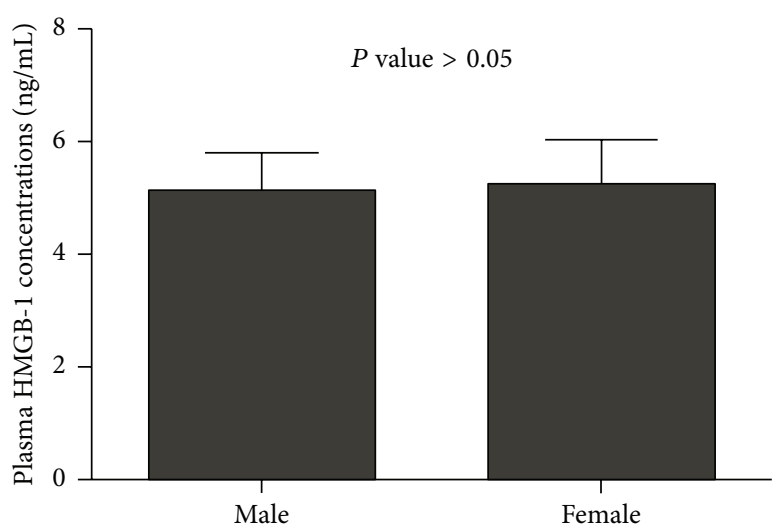

(d)

Figure 1: Plasma CTRP-3 (a) and HMGB-1 (b) concentrations in different subgroups and in males and females ((c) and (d)). Data were presented as means \pm standard deviation. Differences between multiple groups were tested by analysis of variance (ANOVA) for continuous variables. $P$ for trend was estimated by a linear-by-linear association of the chi-square test. ${ }^{*} P<0.05$ compared with NGT, ${ }^{\dagger} P<0.05$ compared with pre-DM. CTRP-3, Clq/TNF-related protein-3; HMGB-1, high-mobility group box-1; NGT, normal glucose tolerance; pre$\mathrm{DM}$, prediabetes states (including IFG, impaired fasting glucose; IGT, impaired glucose tolerance; IGR, impaired glucose regulation); T2DM, type 2 diabetes.

while the proportion of nT2DM was 56.4, 14.7, and 6.3\%, respectively $(X 2=35.607, P$ trend $<0.001)$.

Partial correlation analyses adjusted by age and gender showed that plasma CTRP-3 was negatively correlated with FPG, 2 h PG, FINS, HOMA-IR, HbAlc, BMI, WHR, TG, LDL-C, and IL-6 and was positively associated with HDLC (all $P<0.05$ ). However, after controlling for age and sex, plasma HMGB-1 was positively correlated with variables including FPG, $2 \mathrm{~h}$ PG, FINS, HOMA-IR, HbAlc, BMI, WHR, TG, LDL-C, and IL-6 and was negatively associated with HDL-C (all $P<0.05$ ) (Table 2). Further multiple linear regression analyses showed that the plasma CTRP-3 concentrations were independently correlated with HOMAIR $(\beta=-0.247, P=0.009)$, HbAlc $(\beta=-0.437, P<0.001)$, HDL-C $(\beta=0.144, P=0.048)$, and IL-6 $(\beta=-0.346$, $P<0.001)$. In contrast, plasma HMGB-1 concentrations were independently associated with HOMA-IR ( $\beta=0.297$, $P<0.001)$, IL-6 $(\beta=0.262, P=0.001)$, and creatine $(\beta=0.118, P=0.017)$.
Moreover, further multivariate logistic regression analyses revealed that both plasma CTRP-3 and HMGB-1 concentrations were significantly correlated with pre-DM and nT2DM after controlling for several covariates including age, gender, BMI, WHR, blood pressure, TC, TG, LDL-C, HDL$\mathrm{C}$, and liver and kidney function $(P<0.001$ for all) (Tables 3 and 4).

\section{Discussion}

Recently, Clq/TNF-related protein-3 (CTRP-3) is considered a secreted hormone that plays a role in the hepatic glucose and lipid metabolism. Several researches have reported the association of circulating CTRP-3 with obesity and T2DM in rodent models and in human [13, 26, 27]. Two studies conducted by Peterson et al. [13, 14] showed that both infusion of recombinant CTRP-3 protein and transgenic overexpression of CTRP-3 in mice were involved in regulating the hepatic gluconeogenesis and lipid metabolism. 
TABLE 2: Spearman and partial correlation analyses between variables and plasma CTRP-3 and HMGB-1.

\begin{tabular}{|c|c|c|c|c|c|c|c|c|}
\hline & \multicolumn{2}{|c|}{ Plasma CTRP-3 } & \multicolumn{2}{|c|}{$\begin{array}{c}\text { Plasma CTRP-3 } \\
\text { (age- and sex-adjusted) }\end{array}$} & \multicolumn{2}{|c|}{$\begin{array}{l}\text { Plasma } \\
\text { HMGB-1 }\end{array}$} & \multicolumn{2}{|c|}{$\begin{array}{c}\text { Plasma HMGB-1 } \\
\text { (age- and sex-adjusted) }\end{array}$} \\
\hline & $r$ & $P$ & $r$ & $P$ & $r$ & $P$ & $r$ & $P$ \\
\hline Age (years) & -0.152 & 0.021 & & & 0.106 & 0.111 & & \\
\hline $\mathrm{FPG}(\mathrm{mmol} / \mathrm{L})$ & -0.745 & $<0.001$ & -0.756 & $<0.001$ & 0.615 & $<0.001$ & 0.61 & $<0.001$ \\
\hline INS & -0.635 & $<0.001$ & -0.626 & $<0.001$ & 0.604 & $<0.001$ & 0.529 & $<0.001$ \\
\hline HOMA-IR & -0.763 & $<0.001$ & -0.756 & $<0.001$ & 0.693 & $<0.001$ & 0.689 & $<0.001$ \\
\hline $2 \mathrm{~h} \mathrm{PG}(\mathrm{mmol} / \mathrm{L})$ & -0.67 & $<0.001$ & -0.66 & $<0.001$ & 0.61 & $<0.001$ & 0.604 & $<0.001$ \\
\hline HbAlc (\%) & -0.806 & $<0.001$ & -0.802 & $<0.001$ & 0.677 & $<0.001$ & 0.674 & $<0.001$ \\
\hline BMI $\left(\mathrm{kg} / \mathrm{m}^{2}\right)$ & -0.219 & 0.001 & -0.202 & 0.002 & 0.216 & 0.001 & 0.203 & 0.002 \\
\hline WHR & -0.374 & $<0.001$ & -0.357 & $<0.001$ & 0.255 & $<0.001$ & 0.242 & $<0.001$ \\
\hline SBP (mmHg) & -0.089 & 0.181 & -0.085 & 0.202 & 0.129 & 0.052 & 0.124 & 0.062 \\
\hline $\mathrm{DBP}(\mathrm{mmHg})$ & -0.14 & 0.034 & -0.127 & 0.054 & 0.124 & 0.061 & 0.087 & 0.108 \\
\hline $\mathrm{TC}(\mathrm{mmol} / \mathrm{L})$ & -0.094 & 0.154 & -0.095 & 0.156 & 0.033 & 0.624 & 0.032 & 0.145 \\
\hline $\mathrm{TG}(\mathrm{mmol} / \mathrm{L})$ & -0.213 & 0.001 & -0.188 & 0.004 & 0.163 & 0.014 & 0.145 & 0.029 \\
\hline LDL-C (mmol/L) & -0.152 & 0.021 & -0.142 & 0.032 & 0.25 & $<0.001$ & 0.243 & $<0.001$ \\
\hline HDL-C (mmol/L) & 0.369 & $<0.001$ & 0.372 & $<0.001$ & -0.403 & $<0.001$ & -0.405 & $<0.001$ \\
\hline $\operatorname{ALT}(\mathrm{U} / \mathrm{L})$ & -0.02 & 0.769 & -0.014 & 0.829 & 0.022 & 0.736 & 0.02 & 0.77 \\
\hline AST (U/L) & -0.07 & 0.292 & -0.064 & 0.342 & 0.024 & 0.722 & 0.019 & 0.772 \\
\hline$\gamma$-GGT (U/L) & -0.124 & 0.032 & -0.115 & 0.051 & 0.136 & 0.027 & 0.085 & 0.114 \\
\hline $\mathrm{Cr}$ & -0.056 & 0.4 & -0.055 & 0.41 & 0.066 & 0.324 & 0.067 & 0.319 \\
\hline IL-6 (pg/mL) & -0.797 & $<0.001$ & -0.781 & $<0.001$ & 0.696 & $<0.001$ & 0.695 & $<0.001$ \\
\hline
\end{tabular}

Correlations between variables were analyzed by Spearman's correlation test and age- and sex-adjusted partial correlation test. CTRP-3, Clq/TNF-related protein-3; HMGB-1, high-mobility group box-1; FPG, fasting plasma glucose; FINS, fasting serum insulin; HOMA-IR, homeostasis model assessment for insulin resistance; $2 \mathrm{~h} \mathrm{PG}, 2 \mathrm{~h}$ postchallenge plasma glucose; HbAlc, hemoglobin Alc; BMI, body mass index; WHR, waist-to-hip ratio; SBP, systolic blood pressure; DBP, diastolic blood pressure; TC, total cholesterol; TG, triglyceride; LDL-C, low-density lipoprotein cholesterol; HDL-C, high-density lipoprotein cholesterol; AST, aspartate transaminase; ALT, alanine transaminase; $\gamma$-GGT, gamma-glutamyl transpeptidase; IL-6, interleukin- 6 .

TABLE 3: Multiple logistic regression analyses of plasma CTRP-3 levels among various groups.

\begin{tabular}{|c|c|c|c|c|}
\hline & \multicolumn{2}{|c|}{ Pre-DM } & \multicolumn{2}{|c|}{ nT2DM } \\
\hline & OR, 95\% CI & $P$ value & OR, 95\% CI & $P$ value \\
\hline Age, sex & $0.963(0.952,0.974)$ & $<0.001$ & $0.934(0.92,0.949)$ & $<0.001$ \\
\hline +ALT, AST, GGT, Cr & $0.963(0.952,0.975)$ & $<0.001$ & $0.934(0.919,0.949)$ & $<0.001$ \\
\hline +SBP, DBP & $0.961(0.949,0.973)$ & $<0.001$ & $0.931(0.916,0.947)$ & $<0.001$ \\
\hline +TC, TG, LDL-C, HDL-C & $0.96(0.947,0.973)$ & $<0.001$ & $0.931(0.915,0.949)$ & $<0.001$ \\
\hline +WHR, BMI & $0.961(0.948,0.974)$ & $<0.001$ & $0.933(0.916,0.951)$ & $<0.001$ \\
\hline
\end{tabular}

Pre-DM, prediabetes states (including IFG, impaired fasting glucose; IGT, impaired glucose tolerance; IGR, impaired glucose regulation); T2DM, type 2 diabetes mellitus; OR, odds ratio; CI, confidence interval; ALT, alanine transaminase; AST, aspartate transaminase; $\gamma$-GGT, gamma-glutamyl transpeptidase; $\mathrm{Cr}$, creatinine; SBP, systolic blood pressure; DBP, diastolic blood pressure; TC, total cholesterol; TG, triglyceride; LDL-C, low-density lipoprotein cholesterol; HDL-C, high-density lipoprotein cholesterol; WHR, waist-to-hip ratio; BMI, body mass index.

TABLE 4: Multiple logistic regression analyses of plasma HMGB-1 levels among various groups.

\begin{tabular}{|c|c|c|c|c|}
\hline & \multicolumn{2}{|c|}{ Pre-DM } & \multicolumn{2}{|c|}{ nT2DM } \\
\hline & OR, 95\% CI & $P$ value & OR, 95\% CI & $P$ value \\
\hline Age, sex & $5.436(2.492,11.858)$ & $<0.001$ & $14.436(6.899,26.796)$ & $<0.001$ \\
\hline +ALT, AST, GGT, Cr & $5.142(2.286,11.564)$ & $<0.001$ & $9.81(3.509,15.284)$ & $<0.001$ \\
\hline SBP, DBP & $5.173(2.288,11.696)$ & $<0.001$ & $10.798(4.641,21.068)$ & $<0.001$ \\
\hline TC, TG, LDL-C, HDL-C & $5.505(2.183,13.879)$ & $<0.001$ & $7.805(2.163,17.853)$ & $<0.001$ \\
\hline WHR, BMI & $5.115(1.993,13.125)$ & $<0.001$ & $3.754(1.204,9.281)$ & $<0.001$ \\
\hline
\end{tabular}

Pre-DM, prediabetes states (including IFG, impaired fasting glucose; IGT, impaired glucose tolerance; IGR, impaired glucose regulation); T2DM, type 2 diabetes mellitus; OR, odds ratio; CI, confidence interval; ALT, alanine transaminase; AST, aspartate transaminase; $\gamma$-GGT, gamma-glutamyl transpeptidase; Cr, creatinine; SBP, systolic blood pressure; DBP, diastolic blood pressure; TC, total cholesterol; TG, triglyceride; LDL-C, low-density lipoprotein cholesterol; HDL-C, high-density lipoprotein cholesterol; WHR, waist-to-hip ratio; BMI, body mass index. 
Recombinant CTRP-3 administration overtly lowered the blood glucose levels in C57BL/6 mice and leptin-deficient obese (ob/ob) mice [13]. In our study, we found plasma CTRP-3 concentrations were significantly lower in subjects with pre-DM and nT2DM compared with the NGT group. A multiple linear regression analysis showed the plasma CTRP3 levels were independently associated with insulin resistance, HbAlc, and HDL-C. Further multiple logistical analyses indicated that plasma CTRP-3 concentrations were significantly correlated with pre-DM and nT2DM after adjusting for potential confounders. These data manifest that CTRP-3 is an independent and strong predictor for prediabetes and diabetes. However, our study was contrary to Choi et al. [15] who reported higher CTRP-3 concentrations in pre-DM and T2DM.

The potential mechanism of the antiglucose effect of CTRP-3 remains unknown. In mice, increased CTRP-3 may activate the Akt signaling pathway in the liver while simultaneously suppressing the hepatic gluconeogenic gene expression, since CTRP-3 administration suppressed G6Pase and PEPCK (two main gluconeogenic enzymes) by $80 \%$ in the murine liver [13]. However, recent study by Wolf et al. [26] found no significant change in the whole body glucose metabolism, insulin sensitivity, and fasting-induced hepatic gluconeogenesis in CTRP-3-deficient mice treated with LFD or HFD. Conflicting results also remain among several clinical studies regarding the association between circulating CTRP-3 and T2DM [15-17].

Furthermore, recent data uncovers the anti-inflammatory properties of CTRP-3 in various models in vivo [28, 29], in vitro [30], and ex vivo [31]. CTRP-3-deficient mice showed remarkably deteriorated inflammatory joint pathology in a collagen-induced rheumatoid arthritis model [28]. In CTRP3 knock-out mice, high-fat diet suppressed its liver and adipose expression of profibrotic TGF $\beta 1$ and serum TGF $\beta 1$ concentrations, while greatly increasing serum IL-6 levels [26]. Previously, Schmid et al. [29] reported recombinant CTRP-3 administration could attenuate the systemic inflammation in wild-type mice challenged with a sublethal dose of bacterial-derived lipopolysaccharide (LPS). Contrary to this report, Petersen et al. [32] found neither overexpression nor deficiency of CTRP-3 could affect circulating IL-1b, IL-6, or TNF- $\alpha$ levels in acute LPS-challenged mice. Moreover, highfat feeding is considered a chronic low-grade inflammatory state. In high-fat fed mice, overexpression of CTRP-3 reduced the proinflammatory cytokines such as serum IL-5 and TNF$\alpha$ and elevated soluble gp130 (sgp130) levels [32]. Soluble gp130 is known to antagonize the inflammatory responses by binding to the cytokines of IL-6 family [33], and its serum levels are higher in older individuals with metabolic syndrome [34]. TNF- $\alpha$ is a potent inducer of insulin resistance [35], whereas IL-5 can activate the eosinophil cells to participate in inflammation [36]. In high-fat fed CTRP-3transgenic mice, the attenuated systemic inflammation was accompanied with improved insulin sensitivity [14]. In our study, plasma IL- 6 concentrations were significantly higher in subjects with pre-DM and nT2DM, and the multiple linear regression analyses also showed that IL- 6 was an independent risk factor for CTRP-3. Though the causality cannot be concluded, our results suggest that CTRP-3 may participate in the pathogenesis of inflammation mediated diabetes.

Conventionally, HMGB-1 was considered as a nuclear protein to regulate gene transcription. It was not until 1999 that the cytosolic HMGB-1 was found to be a proinflammatory mediator and can be secreted by activated macrophages in the context of infection, injury, or other inflammatory status [37]. There are several pathways by which HMGB-1 can induce inflammation. First, HMGB-1 can upregulate the advanced glycation end-product (RAGE) signaling by binding to the RAGE receptor, thus boosting inflammation response [38]. Second, HMGB-1 can activate the nuclear factor- $\kappa \mathrm{B}(\mathrm{NF}-\kappa \mathrm{B})$ in metabolic disease [21]. In cells, activated NF- $\kappa \mathrm{B}$ enables the biosynthesis of several proinflammatory mediators such as tumor necrosis factor- $\alpha$ (TNF- $\alpha$ ), interleukin-6 (IL-6), and IL-1 $\beta$, which participate in the pathological inflammatory response [39]. In addition, HMGB-1 can bind to the Toll-like receptor 2 (TLR2) and TLR4. As a classical innate immunity pathway, the TLRs can affect the development and progression of diabetes through the NF- $\kappa \mathrm{B}$ signaling [40]. Through MyD88 signaling, activated TLR2 and TLR4 can trigger the release of proinflammatory factors such as IL-6, TNF- $\alpha$, and IL- $1 \beta$ and the aggregation of inflammatory cells [41]. Inflammatory microenvironment could cause $\beta$-cell dysfunction [42] and trigger insulin resistance, which in turn causes gradual progression to T2DM $[43,44]$. As mentioned above, inflammation and insulin resistance already exist in prediabetic stage and are important contributors to the development of diabetes. In our study, we found the plasma HMGB-1 and IL-6 levels were gradually increased across the pre-DM and nT2DM group. Partial correlation and linear regression analyses showed a significant association among plasma HMGB-1, HOMA-IR, and IL-6. Further multiple logistical analyses also demonstrated that plasma HMGB-1 levels were associated with pre-DM and nT2DM after adjusting for several confounders. As reported by Schierbeck et al. [45], circulating HMGB-1 levels were increased in adipocytes from insulin-resistant subjects, while serum HMGB-1 could promote insulin release in INS-1 cells. In a recent study, Giacobbe et al. [46] reported that HMGB-1 was associated with the presence of gestational diabetes mellitus (GDM) and insulin resistance in the third trimester of pregnancy. Insulin resistance and inflammation are involved in the pathogenesis of GDM; women with GDM have higher risk to develop T2DM after pregnancy. Combined with above evidence, we speculate that the increased plasma HMGB-1 concentrations may largely be due to the presence of insulin resistance and the low chronic inflammation in the context of hyperglycemia.

Our study has some limitations. First, this cross-sectional study could not conclude the causality between plasma CTRP-3/HMGB-1 and pre-DM or diabetes; thus a prospective study is required in future research. Second, due to the small sample size in our study, it is important to determine the cut-off value of circulating CTRP-3 and HMGB-1 to predict pre-DM and T2DM in a large sample size including the sex, race, and other potential confounders. Besides, contrary to other studies, we found no significant difference in plasma 
CTRP-3 and HMGB-1 by gender; more researches are needed to clarify the influence by gender.

In conclusion, we found plasma CTRP-3 levels were lower whereas plasma HMGB-1 concentrations were higher in subjects with prediabetes and newly diagnosed T2DM. Circulating CTRP-3 and HMGB-1 concentrations might be promising biomarkers to predict prediabetes and T2DM.

\section{Competing Interests}

The authors declare that they have no conflict of interests.

\section{Authors' Contributions}

Huili Wei and Hua Qu conceived and designed the experiments. Huili Wei, Hua Qu, and Hang Wang performed the experiments. Huili Wei analyzed the data. Huili Wei contributed to the writing of the manuscript.

\section{Acknowledgments}

This study was supported by research grants from the National Key Clinical Specialties Construction Program of China and grants from the Chinese Society of Endocrinology and the National Natural Science Foundation of China (nos. 81270911, 81070639, 30771038, and 30570744).

\section{References}

[1] L. Guariguata, D. R. Whiting, I. Hambleton, J. Beagley, U. Linnenkamp, and J. E. Shaw, "Global estimates of diabetes prevalence for 2013 and projections for 2035," Diabetes Research and Clinical Practice, vol. 103, no. 2, pp. 137-149, 2014.

[2] J. Beagley, L. Guariguata, C. Weil, and A. A. Motala, "Global estimates of undiagnosed diabetes in adults," Diabetes Research and Clinical Practice, vol. 103, no. 2, pp. 150-160, 2014.

[3] K. G. M. M. Alberti and P. Z. Zimmet, "Definition, diagnosis and classification of diabetes mellitus and its complications. Part 1: diagnosis and classification of diabetes mellitus. Provisional report of a WHO consultation," Diabetic Medicine, vol. 15, no. 7, pp. 539-553, 1998.

[4] M. Kanat, L. Norton, D. Winnier, C. Jenkinson, R. A. Defronzo, and M. A. Abdul-Ghani, "Impaired early- but not late-phase insulin secretion in subjects with impaired fasting glucose," Acta Diabetologica, vol. 48, no. 3, pp. 209-217, 2011.

[5] M. A. Abdul-Ghani, C. P. Jenkinson, D. K. Richardson, D. Tripathy, and R. A. DeFronzo, "Insulin secretion and action in subjects with impaired fasting glucose and impaired glucose tolerance: results from the veterans administration genetic epidemiology study," Diabetes, vol. 55, no. 5, pp. 1430-1435, 2006.

[6] V. Grossmannm, V. H. Schmitt, T. Zeller et al., "Profile of the immune and inflammatory response in individuals with prediabetes and type 2 diabetes," Diabetes Care, vol. 38, no. 7, pp. 1356-1364, 2015.

[7] A. G. Tabak, C. Herder, W. Rathmann et al., "Prediabetes: a high-risk state for diabetes development," The Lancet, vol. 379, no. 9833, pp. 2279-2290, 2012.

[8] R. R. Giraldez, R. M. Clare, R. D. Lopes et al., "Prevalence and clinical outcomes of undiagnosed diabetes mellitus and prediabetes among patients with high-risk non-ST-segment elevation acute coronary syndrome," American Heart Journal, vol. 165, no. 6, pp. 918-925.e2, 2013.

[9] R. B. Stacey, P. E. Leaverton, D. D. Schocken, J. A. Peregoy, and A. G. Bertoni, "Prediabetes and the association with unrecognized myocardial infarction in the multi-ethnic study of atherosclerosis," American Heart Journal, vol. 170, no. 5, pp. 923-928, 2015.

[10] W. Yang, J. Lu, J. Weng et al., "Prevalence of diabetes among men and women in China," The New England Journal of Medicine, vol. 362, no. 12, pp. 1090-1101, 2010.

[11] T. Maeda, M. Abe, K. Kurisu, A. Jikko, and S. Furukawa, "Molecular cloning and characterization of a novel gene, CORS26, encoding a putative secretory protein and its possible involvement in skeletal development," The Journal of Biological Chemistry, vol. 276, no. 5, pp. 3628-3634, 2001.

[12] G. W. Wong, S. A. Krawczyk, C. Kitidis-Mitrokostas, T. Revett, R. Gimeno, and H. F. Lodish, "Molecular, biochemical and functional characterizations of Clq/TNF family members: adiposetissue-selective expression patterns, regulation by PPAR- $\gamma$ agonist, cysteine-mediated oligomerizations, combinatorial associations and metabolic functions," Biochemical Journal, vol. 416, no. 2, pp. 161-177, 2008.

[13] J. M. Peterson, Z. Wei, and G. W. Wong, "Clq/TNF-related protein-3 (CTRP3), a novel adipokine that regulates hepatic glucose output," The Journal of Biological Chemistry, vol. 285, no. 51, pp. 39691-39701, 2010.

[14] J. M. Peterson, M. M. Seldin, Z. Wei, S. Aja, and G. W. Wong, "CTRP3 attenuates diet-induced hepatic steatosis by regulating triglyceride metabolism," American Journal of PhysiologyGastrointestinal and Liver Physiology, vol. 305, no. 3, pp. G214G224, 2013.

[15] K. M. Choi, S. Y. Hwang, H. C. Hong et al., "Clq/TNF-related protein-3 (CTRP-3) and pigment epithelium-derived factor (PEDF) concentrations in patients with type 2 diabetes and metabolic syndrome," Diabetes, vol. 61, no. 11, pp. 2932-2936, 2012.

[16] R. M. Wolf, K. E. Steele, L. A. Peterson, T. H. Magnuson, M. A. Schweitzer, and G. W. Wong, "Lower circulating C1q/TNFrelated protein-3 (CTRP3) levels are associated with obesity: a cross-sectional study," PLoS ONE, vol. 10, no. 7, Article ID e0133955, 2015.

[17] H. Qu, M. Deng, H. Wang et al., "Plasma CTRP-3 concentrations in Chinese patients with obesity and type II diabetes negatively correlate with insulin resistance," Journal of Clinical Lipidology, vol. 9, no. 3, pp. 289-294, 2015.

[18] P. S. Petersen, R. M. Wolf, X. Lei et al., "Immunomodulatory roles of CTRP3 in endotoxemia and metabolic stress," Physiological Reports, vol. 4, no. 5, Article ID e12735, 2016.

[19] J. Weigert, M. Neumeier, A. Schäffler et al., "The adiponectin paralog CORS-26 has anti-inflammatory properties and is produced by human monocytic cells," FEBS Letters, vol. 579, no. 25, pp. 5565-5570, 2005.

[20] G. H. Goodwin, C. Sanders, and E. W. Johns, "A new group of chromatin-associated proteins with a high content of acidic and basic amino acids," European Journal of Biochemistry, vol. 38, no. 1, pp. 14-19, 1973.

[21] J. R. van Beijnum, W. A. Buurman, and A. W. Griffioen, "Convergence and amplification of toll-like receptor (TLR) and receptor for advanced glycation end products (RAGE) signaling pathways via high mobility group B1 (HMGB1)," Angiogenesis, vol. 11, no. 1, pp. 91-99, 2008. 
[22] R. Guzmán-Ruiz, F. Ortega, A. Rodríguez et al., "Alarmin highmobility group B1 (HMGB1) is regulated in human adipocytes in insulin resistance and influences insulin secretion in $\beta$-cells," International Journal of Obesity, vol. 38, no. 12, pp. 1545-1554, 2014.

[23] H. Wang, H. Qu, and H. Deng, "Plasma HMGB-1 levels in subjects with obesity and type 2 diabetes: a cross-sectional study in China," PLoS ONE, vol. 10, no. 8, Article ID e0136564, 2015.

[24] Y. Chen, F. Qiao, Y. Zhao, Y. Wang, and G. Liu, "HMGB1 is activated in type 2 diabetes mellitus patients and in mesangial cells in response to high glucose," International Journal of Clinical and Experimental Pathology, vol. 8, no. 6, pp. 66836691, 2015.

[25] American Diabetes Association, "Diagnosis and classification of diabetes mellitus," Diabetes Care, vol. 37, no. 1, pp. S81-S90, 2014.

[26] R. M. Wolf, X. Lei, Z. C. Yang, M. Nyandjo, S. Y. Tan, and G. W. Wong, "CTRP3 deficiency reduces liver size and alters IL-6 and TGF $\beta$ levels in obese mice," American Journal of PhysiologyEndocrinology and Metabolism, vol. 310, no. 5, pp. E332-E345, 2016.

[27] B. Ban, B. Bai, M. Zhang et al., "Low serum cartonectin/CTRP3 concentrations in newly diagnosed type 2 diabetes mellitus: in vivo regulation of cartonectin by glucose," PLOS ONE, vol. 9, no. 11, Article ID e112931, 2014.

[28] M. A. Murayama, S. Kakuta, T. Maruhashi et al., "CTRP3 plays an important role in the development of collagen-induced arthritis in mice," Biochemical and Biophysical Research Communications, vol. 443, no. 1, pp. 42-48, 2014.

[29] A. Schmid, A. Kopp, F. Hanses, T. Karrasch, and A. Schäffler, "Clq/TNF-related protein-3 (CTRP-3) attenuates lipopolysaccharide (LPS)-induced systemic inflammation and adipose tissue Erk-1/-2 phosphorylation in mice in vivo," Biochemical and Biophysical Research Communications, vol. 452, no. 1, pp. 8-13, 2014.

[30] A. Kopp, M. Bala, C. Buechler et al., "Clq/TNF-related protein3 represents a novel and endogenous lipopolysaccharide antagonist of the adipose tissue," Endocrinology, vol. 151, no. 11, pp. 5267-5278, 2010.

[31] A. Kopp, M. Bala, J. Weigert et al., "Effects of the new adiponectin paralogous protein CTRP-3 and of LPS on cytokine release from monocytes of patients with type 2 diabetes mellitus," Cytokine, vol. 49, no. 1, pp. 51-57, 2010.

[32] P. S. Petersen, R. M. Wolf, X. Lei et al., "Immunomodulatory roles of CTRP3 in endotoxemia and metabolic stress," Physiological Reports, vol. 4, no. 5, Article ID 12735, 2016.

[33] J. S. Silver and C. A. Hunter, "gp130 at the nexus of inflammation, autoimmunity, and cancer," Journal of Leukocyte Biology, vol. 88, no. 6, pp. 1145-1156, 2010.

[34] G. Zuliani, M. Galvani, M. Maggio et al., "Plasma soluble gp130 levels are increased in older subjects with metabolic syndrome. The role of insulin resistance," Atherosclerosis, vol. 213, no. 1, pp. 319-324, 2010.

[35] G. S. Hotamisligil, N. S. Shargill, and B. M. Spiegelman, "Adipose expression of tumor necrosis factor- $\alpha$ : direct role in obesity-linked insulin resistance," Science, vol. 259, no. 5091, pp. 87-91, 1993.

[36] T. Kouro and K. Takatsu, "IL-5- and eosinophil-mediated inflammation: from discovery to therapy," International Immunology, vol. 21, no. 12, pp. 1303-1309, 2009.
[37] H. Wang, O. Bloom, M. Zhang et al., "HMG-1 as a late mediator of endotoxin lethality in mice," Science, vol. 285, no. 5425, pp. 248-251, 1999.

[38] O. Hori, J. Brett, T. Slattery et al., "The receptor for advanced glycation end products (RAGE) is a cellular binding site for amphoterin. Mediation of neurite outgrowth and co-expression of rage and amphoterin in the developing nervous system," The Journal of Biological Chemistry, vol. 270, no. 43, pp. 2575225761, 1995.

[39] J. S. Park, J. Arcaroli, H.-K. Yum et al., "Activation of gene expression in human neutrophils by high mobility group box 1 protein," American Journal of Physiology-Cell Physiology, vol. 284, no. 4, pp. C870-C879, 2003.

[40] F. S. Wong and L. Wen, "Toll-like receptors and diabetes," Annals of the New York Academy of Sciences, vol. 1150, pp. 123132, 2008.

[41] C.-S. Yang, D.-M. Shin, H.-M. Lee et al., "ASK1-p38 MAPKp47phox activation is essential for inflammatory responses during tuberculosis via TLR2-ROS signalling," Cellular Microbiology, vol. 10, no. 3, pp. 741-754, 2008.

[42] J. Yin, Y. Peng, J. Wu, Y. Wang, and L. Yao, “Toll-like receptor $2 / 4$ links to free fatty acid-induced inflammation and $\beta$-cell dysfunction," Journal of Leukocyte Biology, vol. 95, no. 1, pp. 4752, 2014.

[43] G. S. Hotamisligil, "Inflammation and metabolic disorders," Nature, vol. 444, no. 7121, pp. 860-867, 2006.

[44] M. Y. Donath and S. E. Shoelson, "Type 2 diabetes as an inflammatory disease," Nature Reviews Immunology, vol. 11, no. 2, pp. 98-107, 2011.

[45] H. Schierbeck, H. Wähämaa, U. Andersson, and H. E. Harris, "Immunomodulatory drugs regulate HMGB1 release from activated human monocytes," Molecular Medicine, vol. 16, no. 9-10, pp. 343-351, 2010.

[46] A. Giacobbe, R. Granese, R. Grasso et al., "Association between maternal serum high mobility group box 1 levels and pregnancy complicated bygestational diabetes mellitus," Nutrition, Metabolism and Cardiovascular Diseases, vol. 26, no. 5, pp. 414418, 2016. 


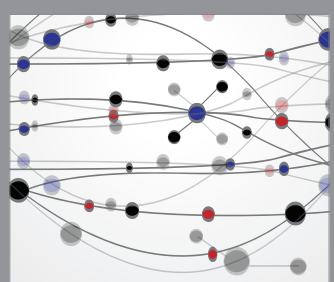

The Scientific World Journal
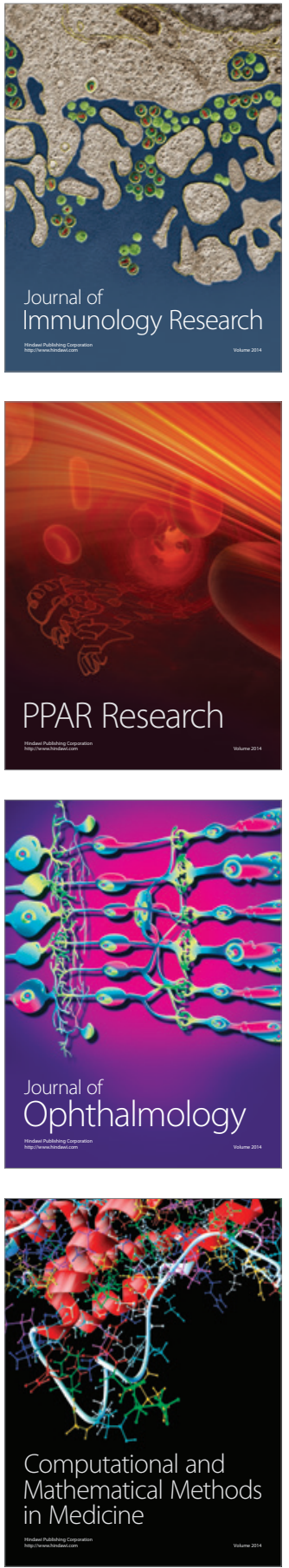

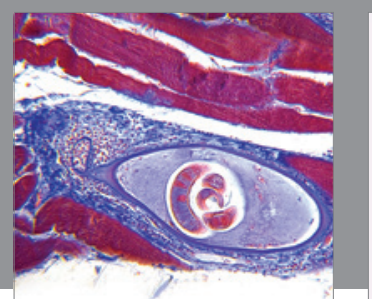

Gastroenterology Research and Practice

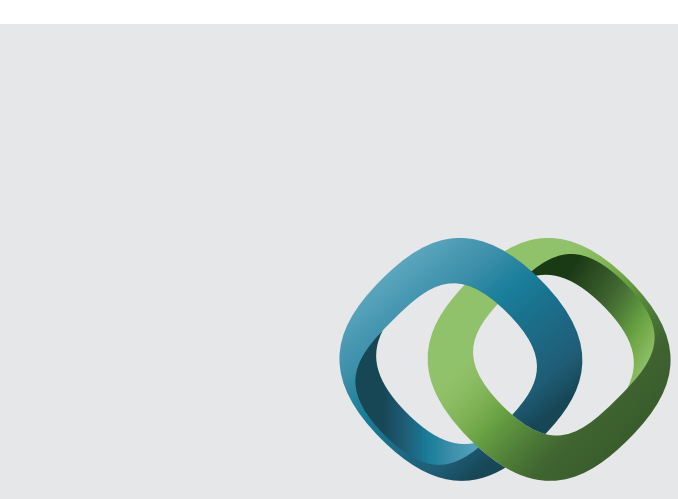

\section{Hindawi}

Submit your manuscripts at

http://www.hindawi.com
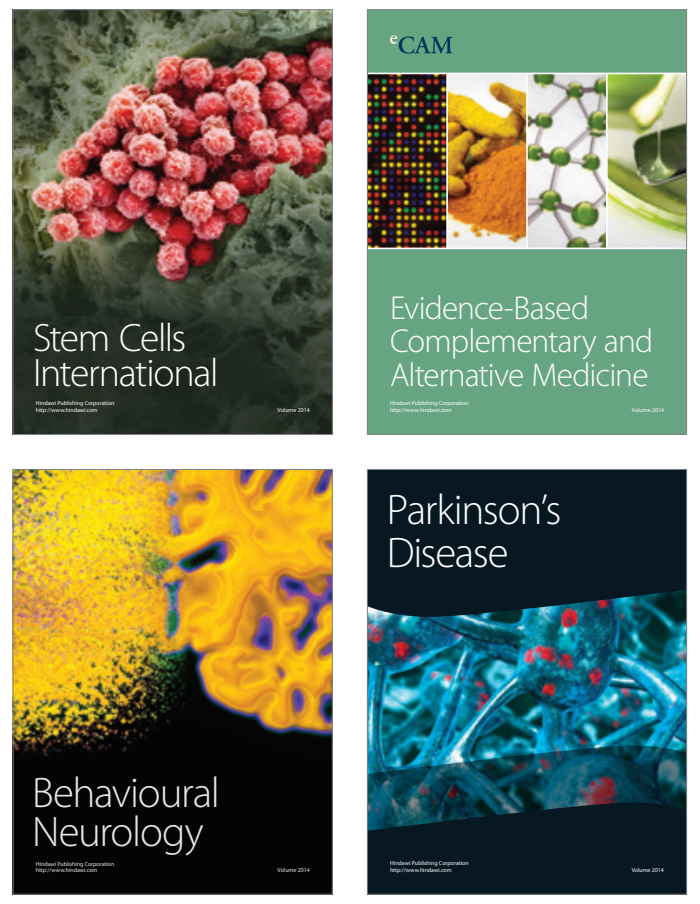
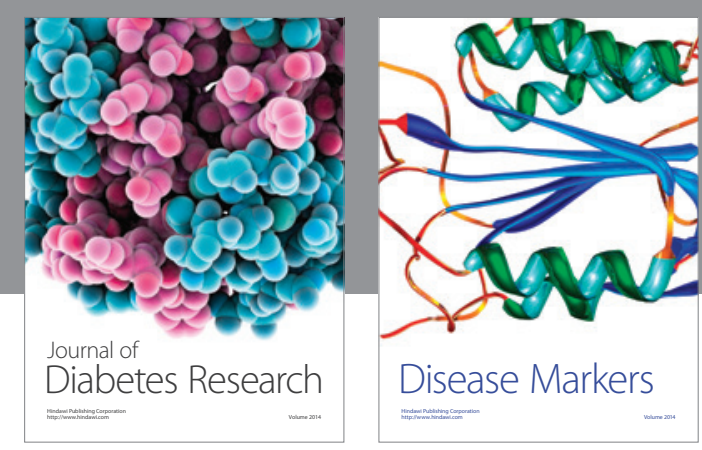

Disease Markers
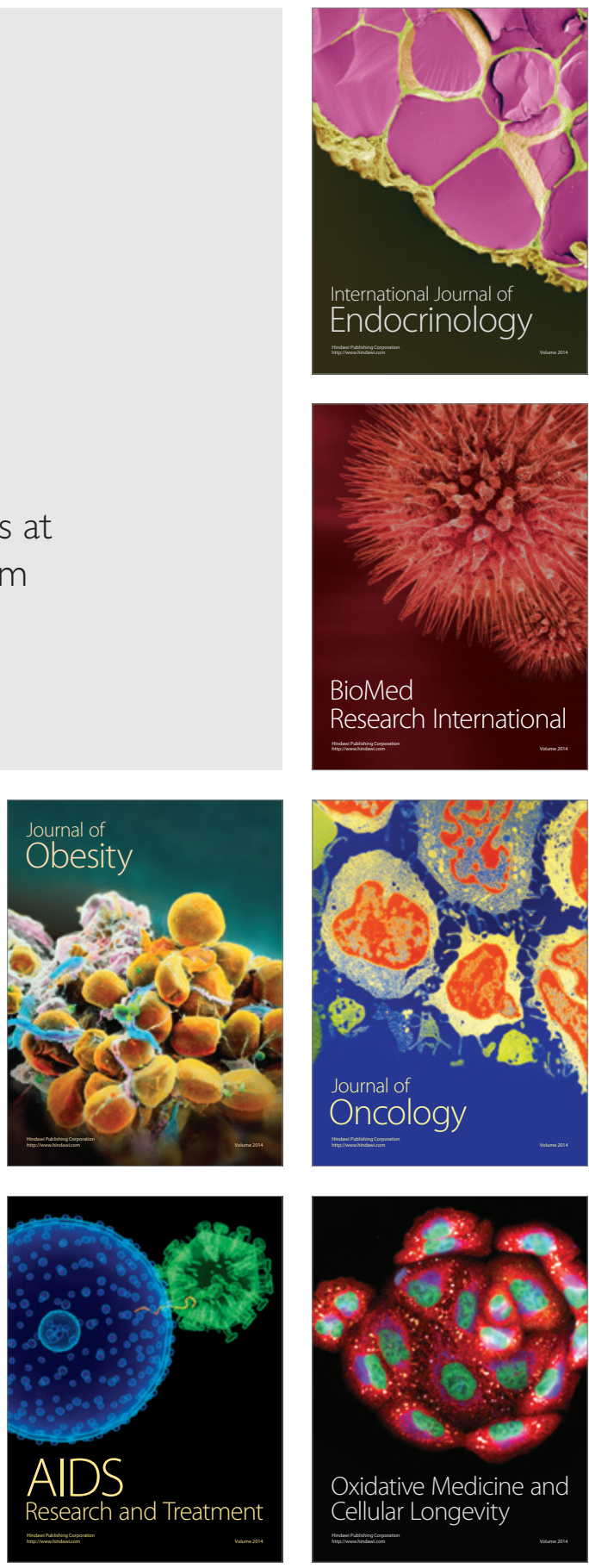